\title{
Genomic analysis of bovine respiratory disease and lung consolidation in preweaned Holstein calves using clinical scoring and lung ultrasound
}

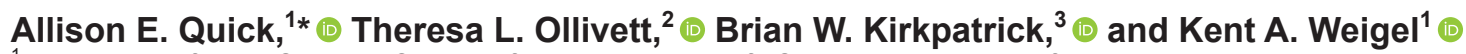 \\ ${ }^{1}$ Department of Dairy Science, College of Agricultural and Life Sciences, University of Wisconsin, Madison 53706 \\ ${ }^{2}$ Department of Medical Sciences, School of Veterinary Medicine, University of Wisconsin, Madison 53706 \\ ${ }^{3}$ Department of Animal Sciences, College of Agricultural and Life Sciences, University of Wisconsin, Madison 53706
}

\begin{abstract}
Bovine respiratory disease $(\mathrm{BRD})$ is a leading cause of morbidity and mortality in dairy calves, with detrimental long-term effects that include stunted growth, increased age at first calving, and decreased milk production in first lactation. The objectives of this study were to establish a protocol for objective and efficient assessment of BRD phenotypes in preweaned dairy calves, develop a genomic reference population with well-defined clinical and subclinical phenotypes, identify chromosomal regions associated with BRD in a genome-wide association study, estimate genetic parameters of $\mathrm{BRD}$, and predict genomic breeding values of dairy calves. A total of 1,107 Holstein calves from 6 dairy farms in southern Wisconsin were examined using clinical respiratory scoring and lung ultrasound at 3 and 6 wk of age. The clinical respiratory score was based on visual appraisal of eyes, nose, ears, cough, and temperature. Lung ultrasound scores were assigned based on the amount of consolidation present. Calves were genotyped with a commercially available SNP array and after quality control and imputation to higher density, 690,291 SNP markers and 1,014 individuals remained. Single-step genome-wide association study and single-step genomic best linear unbiased prediction were applied to binary 3- and 6-wk phenotypes considered as overall respiratory healthy versus affected (RESP3, RESP6) or as presence or absence of lung consolidation (CON3, CON6). Lung ultrasound combined with a clinical scoring system allowed for efficient and objective assessment for the prevalence of BRD. Proportions of variance attributed to $1-\mathrm{Mb}$ non-overlapping windows suggested genomic regions that may contain putative candidate genes, most notably regions on Bos taurus autosomes 1, 6, 7, 10,11,12, 15, 17, 18, 27, and 28 that explained 0.70 to $1.45 \%$ of the genetic variance. Heritability estimates were higher at $3 \mathrm{wk}(0.214$ and
\end{abstract}

Received February 22, 2019.

Accepted October 4, 2019.

*Corresponding author: aquick2@wisc.edu
0.241 for CON3 and RESP3, respectively) than $6 \mathrm{wk}$ (0.084 and 0.111 for CON6 and RESP6, respectively), and mean reliabilities of genomic estimated breeding vales for calves with genotypes and phenotypes ranged from 0.12 for CON6 to 0.30 for RESP3.

Key words: genome-wide association study, reference population, bovine respiratory disease, heritability

\section{INTRODUCTION}

Bovine respiratory disease (BRD) is a common cause of morbidity and mortality in US dairy calves, reportedly affecting $12 \%$ of preweaned calves in a National Animal Health Monitoring System survey of commercial dairy farms (USDA, 2018) and $18 \%$ of preweaned calves in a National Animal Health Monitoring System survey of custom dairy heifer rearing operations (USDA, 2012). Although reducing the incidence of BRD through improved management and prevention has been a focus for more than 2 decades, incidence rates have remained steady (i.e., USDA, 2010, 2018). The prevention, treatment, and death loss cost associated with BRD has been estimated as $\$ 9.84$ to 16.35 per preweaned calf and $\$ 2.05$ to 2.22 per postweaned calf (Gorden and Plummer, 2010). In addition, van der Fels-Klerx et al. (2001) noted that BRD can impair growth and increase mortality risk, both at the time of disease and later in life. Lung consolidation and BRD in calves have been associated with a $525-\mathrm{kg}$ decrease in milk production during first lactation, as well as increased age at first calving and increased likelihood of dystocia (Stanton et al., 2012; Teixeira et al., 2017; Dunn et al., 2018).

Subjectivity in diagnosing a disease and evaluating its severity complicates genetic selection and management for improved animal health. Additionally, subclinical cases of BRD are not detected with traditional scoring systems and further complicate accurate classification of diseased calves (Ollivett et al., 2015). Keele et al. (2015) carried out a genome-wide association study (GWAS) of BRD in beef cattle by evaluating lung lesions detected at the time of slaughter, which is a 
commonly used method for identifying beef cattle with pneumonia. They identified 14 SNP markers on 10 chromosomes that were significant at a $5 \%$ genomewide error rate; these were a subset of 85 SNP markers on 28 chromosomes that were significant at a $5 \%$ false discovery rate, leading the authors to conclude that the genomic footprint of lung lesions is highly complex. Neibergs et al. (2014) used a case-control design and coupled clinical respiratory scoring (CRS) with deep pharyngeal and mid-nasal diagnostic swabs of Holstein calves from California and New Mexico. Four different GWAS methods were used, and concordance was observed between SNP genotypes and BRD, but the chromosomal locations most closely associated with BRD phenotypes differed by state. Producer-recorded treatment data, including more than 2 million BRD phenotypes of Holstein calves, were used in a recent study by Vukasinovic et al. (2018). Single-step GWAS (ssGWAS) with a 10-SNP sliding window implicated regions of BTA1, 2, 9, 14, and 27, as well as the $\mathrm{X}$ chromosome, that were associated with BRD during the first year of life.

Studies based on the presence or absence of lung lesions in a postmortem examination can be informative; however, they tend to be limited to few animals and rarely include dairy breeds. Studies using farmerrecorded health events or treatment rates are affected by misdiagnosis and underreporting (Sivula et al., 1996). Gulliksen et al. (2009) indicated that the incidence of BRD in Norwegian dairy herds may have been underestimated by $40 \%$ due to underreporting by dairy farmers, and Buczinski et al. (2014) noted that farmerrecorded data had sensitivity of $71.4 \%$ to detect lung consolidation. Scoring systems to identify calves with BRD have been developed, based on varying parameters and levels of severity; for example, McGuirk and Peek (2014) described the Wisconsin CRS system, which provided $62 \%$ sensitivity and $74 \%$ specificity in a Bayesian analysis (Buczinski et al., 2015). Methods such as deep pharyngeal swabs identify pathogens present with high sensitivity and specificity, but they are invasive and labor intensive. Ollivett et al. (2015) and Buczinski et al. (2015) described and validated a method for assessment of lung lesions in dairy calves based on lung ultrasound score (LUS). The sensitivity and specificity of lung ultrasound (LU) for detection of lung lesions associated with subclinical respiratory disease were 94 and $100 \%$, respectively, when postmortem evaluation of lung lesions was used as the diagnostic gold standard (Ollivett et al., 2015). This method is noninvasive and performs consistently inter- and intraobserver, allowing for an objective analysis (Buczinski et al., 2013).

As an industry, we have focused on reducing the environmental risk factors through housing, management, and vaccinations that may improve respiratory health, but cannot completely eliminate diseases. However, genetic selection has the potential to confer permanent improvement in resistance or resilience to BRD infection and has not been fully explored at this point in time.

Whole-genome (genomic) selection has revolutionized animal breeding, and selection of animals that are more resistant to infection or resilient to development of lung lesions using genomic estimated breeding values (GEBV) could be a long-term solution to reducing the incidence of BRD. Genomic selection allows early and accurate selection decisions for traits that are difficult to measure routinely. Once a reference population of animals with well-defined phenotypes and SNP genotypes has been established, GEBV of young selection candidates can be predicted from associations between SNP genotypes and trait phenotypes observed in the reference population. But it is essential to refresh phenotypes and continue to expand the reference population to accurately represent changes in complex traits and reflect the genetic progress seen in population and selection candidates.

An early study that used daily veterinary visits to determine BRD status evaluated the relationship between genetics and BRD in preweaned beef calves (Snowder et al., 2005). Pedigree-based estimates of heritability were 0.20 on the observed scale and 0.48 when converted to an underlying liability scale. This indicated that substantial variation exists in BRD susceptibility if animals are exposed to causative pathogens. In a later study, Buchanan et al. (2016) reported heritability estimates of 0.14 and 0.15 for binary and ordered categorical definitions of BRD in Charolais steers under feedlot conditions.

In dairy calves, Heringstad et al. (2008) reported a heritability estimate of 0.05 on the underlying liability scale for the incidence of BRD in Norwegian Red calves from birth to 180 d of age. Henderson et al. (2011) reported heritability estimates of 0.09 for $\mathrm{BRD}, 0.14$ for umbilical diseases, and 0.04 for bloat in Holstein calves from 1 to $7 \mathrm{~d}$ of age. In the aforementioned case-control study of Holstein calves in California and New Mexico (Neibergs et al., 2014), heritability estimates from genomic BLUP were 0.20 and 0.22 for New Mexico and California, respectively, and 0.13 in the combined analysis.

Heritability estimates for BRD in Holstein calves in the study Vukasinovic et al. (2018) were 0.10 at 0 to 3 d of age, 0.06 at 4 to $14 \mathrm{~d}$ of age, 0.05 at 15 to $50 \mathrm{~d}$ of age, and 0.04 at 51 to $365 \mathrm{~d}$ of age. Mean reliabilities of GEBV for young selection candidates without progeny or phenotypes ranged from $38 \%$ at 51 to $365 \mathrm{~d}$ of age to $50 \%$ at 0 to $3 \mathrm{~d}$ of age, and results have been added to 
the CLARIFIDE Plus portfolio of genomic predictions offered routinely by Zoetis (Kalamazoo, MI), along with predictions for scours and livability (GonzalezPeña et al., 2019).

To date, none of the genetic studies have used LU to increase the precision of classifying BRD phenotypes. Lung ultrasound is a highly sensitive and specific diagnostic tool that allows objective identification of calves with clinical or subclinical BRD, while providing an assessment of the severity of lung consolidation. Therefore, the objectives of this study were to create a deeply phenotyped reference population of Holstein calves using clinical scoring and LUS, search for associations of specific SNP and genomic regions with BRD and lung consolidation, estimate genetic parameters of $\mathrm{BRD}$ and lung consolidation, and predict GEBV that will inform future development of a selection program.

\section{MATERIALS AND METHODS}

Data collection for this cohort study took place at 6 commercial dairy farms located in southern Wisconsin from May to August 2017. Farms were selected based on large herd size, distance from the UW-Madison campus (less than 70 miles or $112.6 \mathrm{~km}$ ), and routine genomic testing of all heifer calves. The Institutional Animal Care and Use Committee at the University of Wisconsin-Madison (\#A005049-R03) approved this study.

\section{Phenotypic Data}

Four trained evaluators visited each farm weekly. Evaluators completed LUS training by Ollivett following protocol laid out by Ollivett and Buczinski (2016) to ensure homogeneous assessment. All female Holstein calves born during the study period were enrolled at approximately 3 wk old. At enrollment and at approximately 6 wk of age, CRS and LU was performed by a pair of evaluators (McGuirk and Peek, 2014; Ollivett and Buczinski, 2016). Both lungs were examined using LU following application of $100 \mathrm{~mL}$ of $70 \%$ isopropyl alcohol to the hair coat between the first and sixth intercostal spaces. Right middle, caudal, and cranial aspects of the right cranial lobe, as well as left caudal and cranial aspects of the left cranial lobe, were assessed using an IBEX Pro scanner (E.I. Medical, Loveland, $\mathrm{CO})$ with a linear rectal transducer as described by Ollivett and Buczinski (2016). Ultrasonographic gains were set at 18, 25, and $36 \mathrm{~dB}$ for the overall, near field, and far fields, respectively. All CRS and LU data were entered into the University of Wisconsin-Madison Calf Health Scorer iPad application (https://www.vetmed .wisc.edu/dms/fapm/apps/chs.htm). A pair of evalua- tors were able to score one calf every 2 to $5 \mathrm{~min}$. Calves that scored $\geq 2$ for at least 2 categories were considered CRS $(+)$ (McGuirk and Peek, 2014). Calves with $\geq 1 \mathrm{~cm}$ lung consolidation were considered $\mathrm{LU}(+)$ (Ollivett et al., 2015). All scorers were blinded to genomic status of the calf.

\section{Genomic Data}

Genomic testing was carried out by Zoetis (Kalamazoo, MI) using the ZL5 SNP array, with genotypes for 35,334 SNP from calves on 5 farms. Quality control was carried out in PLINK 1.90b5.2 (Purcell et al., 2007) with default criteria for removal of observations with genotype or individual call rate $<0.95$, minor allele frequency $<0.05$, or Hardy-Weinberg equilibrium $<1 \mathrm{E}$ 6; 1,014 animals and 28,696 SNP remained after editing. Imputation to higher density, specifically 690,291 SNP markers, utilized BEAGLE 4.1 (Browning and Browning, 2016) with a reference population of 746 US Holstein cows previously genotyped with Illumina (San Diego, CA). BovineHD and GeneSeek (Lincoln, NE) Genomic Profiler GGP F-250 chips. These cows were drawn from 12 commercial herds in Minnesota and Michigan, were sired by $>274$ different sires, and represent a broad sampling from the US Holstein population.

\section{Statistical Analysis}

For each age group (3 vs. 6 wk), phenotypes of respiratory (RESP) and lung consolidation (CON) were defined using 2 methods. For the RESP method, calves that were $\mathrm{CRS}(+), \mathrm{CRS}(+)$ and $\mathrm{LU}(+)$, or $\mathrm{LU}(+)$ were considered $\mathrm{BRD}(+)$, resulting in RESP3 or RESP6 for each age group. For the CON method, only calves that were $\mathrm{LU}(+)$ were considered $\operatorname{BRD}(+)$, resulting in CON3 or CON6 for each age group.

Single-step genomic BLUP (ssGBLUP) and ssGWAS (Aguilar et al., 2010; Wang et al., 2012) were implemented using the BLUPF90 family of programs (RENUMF90 v1.136, AIREMLF90 v1.139, PREGSF90 v1.14, BLUPF90 v1.63, and POSTGSF90 v1.5; Misztal et al., 2015), using a mixed linear univariate model with herd as a fixed effect and animal additive genetic and residual as random effects. A linear model was chosen because the 3 -wk data sets would not converge under a threshold model. Pedigree information was used in addition to genotypes in the construction of the relationship matrix. Single-step genomic analysis was selected due to its ease of incorporation of genotypes and pedigrees and ability to run window-variance analysis. Also, because all heifer calves in these herds are subject to routine genomic testing, and because the 
Table 1. Descriptive statistics for the 3- and 6-wk phenotypes, including the number of observations, number genotyped, mean age (d) with SD per time period, and prevalence (\%) for lung consolidation at 3 wk of age (CON3), bovine respiratory disease at 3 wk of age (RESP3), lung consolidation at 6 wk of age (CON6), and bovine respiratory disease at 6 wk of age (RESP6)

\begin{tabular}{lccccc}
\hline Week & $\begin{array}{c}\text { No. of observations } \\
(\mathrm{n}=2,167)\end{array}$ & $\begin{array}{c}\text { No. genotyped } \\
(\mathrm{n}=1,014)\end{array}$ & $\begin{array}{c}\text { Age } \\
(\mathrm{d} ; \text { mean } \pm \mathrm{SD})\end{array}$ & $\begin{array}{c}\text { RESP N } \\
(\%)\end{array}$ & $\begin{array}{c}\text { CON N } \\
(\%)\end{array}$ \\
\hline 3 & 1,102 & 1,007 & $24.25 \pm 2.29$ & $208(18.9)$ & $176(16.0)$ \\
6 & 1,065 & 998 & $45.06 \pm 2.45$ & $244(22.9)$ & $215(20.2)$ \\
\hline
\end{tabular}

advantages of using genomic information in selection and management decisions are well-documented (e.g., Goddard and Hayes, 2007; VanRaden et al., 2009), we decided to perform a genomic analysis only and forego comparisons of genomic and traditional pedigree-based analyses. Birth month was not considered due to the short 10-wk window of data collection. The proportion of variance attributed to each $1-\mathrm{Mb}$ non-overlapping window was derived by dividing the variance of the genetic values for that region by the total genetic variance (Wang et al., 2014), where the former is computed as a product of gene content for individuals at each SNP and the estimated marker effect for the corresponding SNP, summed over all SNP in the window. The top regions of highest proportion of variance (total genetic variance $\geq 0.7 \%$ ) were investigated further. Heritability estimates were derived via AIREMLF90, in conjunction with the single-step analysis.

A multiple-trait version of the ssGBLUP model described above was implemented using AIREMLF90 $\mathrm{v} 1.139$, for the purpose of assessing pairwise genetic correlations by age and trait definition. The 4 pairwise models that were fitted included: RESP3 and CON3, RESP6 and CON6, RESP3 and RESP6, and CON3 and CON6.

Reliabilities (REL) for GEBV of calves in the reference population with phenotypes and genotypes were computed for RESP3, CON3, RESP6, and CON6 phenotypes using prediction error variances $(\mathbf{P E V})$ and the genetic variances $\left(\sigma_{g}^{2}\right)$ as REL $=1-\mathrm{PEV} / \sigma_{g}^{2}$, where $\mathrm{PEV}$ is the squared standard error of the ssGBLUP prediction (Dekkers, 1992; Van Vleck et al., 1992).

\section{RESULTS AND DISCUSSION}

\section{Descriptive Statistics}

In total, 2,167 clinical observations were made on 1,107 calves over a 10 -wk period. Calves were 24.25 \pm 2.29 (mean $\pm \mathrm{SD}) \mathrm{d}$ old at enrollment $(\mathrm{n}=1,102)$ and $45.06 \pm 2.45$ (mean $\pm \mathrm{SD}$ ) d old at their second examination $(\mathrm{n}=1,065)$. Genomic data were initially available on 1,016 calves. After editing, 2 calves were excluded, resulting in a total of 1,014 calves included in the genomic analyses. The 3- and 6-wk prevalences of RESP positive phenotypes were 18.9 and $22.9 \%$, respectively. The 3 - and 6 -wk prevalences of $\mathrm{CON}$ positive phenotypes were 16 and $20.2 \%$, respectively. Table 1 summarizes these results.

The prevalence rate differs between RESP and CON classification groups were $2.9 \%$ for 3 wk and $2.7 \%$ for 6 wk. The observed phenotypic correlation between LUS phenotypes for the same calves at 3 and 6 wk of age was 0.26 , indicating moderate repeatability.

\section{Results of the GWAS}

Four phenotype classifications (RESP3, CON3, RESP6, and CON6) yielded 2,619 non-overlapping windows of $1 \mathrm{Mb}$, with 2 to $601 \mathrm{SNP}$ per window. Figures 1 and 2 show Manhattan plots of proportion of variance attributed to each window in the ssGWAS analysis of 3 and 6 wk phenotypes, respectively. In Figure 1, peaks indicating the largest proportions of variance for CON3 are shown on BTA1, 7, 11, 15, 17, and 18. The largest proportion of variance for CON3 (1.39\% of genetic variance) was on BTA7 from 100,372,315 to $101,365,312 \mathrm{bp}$; this region contains the $R G M B$ gene, which is associated with neural development (Stelzer et al., 2016) and the CHD1 gene, which is associated with body growth and skeletal muscle in cattle (Sadkowski et al., 2009). Results for RESP3, also displayed in Figure 1, show the highest peaks on BTA6, 7, 11, and 17. The largest proportion of variance for RESP3 (1.3\% of genetic variance) and for RESP6 (1.45\% of genetic variance) was on BTA6 in the region of $80,969,240$ to $81,969,240$ bp. This region contains the TECRL gene, which is associated with lipid oxidoreductase (Stelzer et al., 2016). The TECRL was shown previously to have a role in puberty, female fertility, and feed conversion in cattle (de Almeida Santana et al., 2016; Dias et al., 2017). The same significant region that contained TECRL was also discovered for CON6 (Table 3). As shown in Figure 2, BTA6, 10, 12, 27, and 28 contained regions with the largest proportions of variance for CON6, whereas the largest proportions of variance for RESP6 were on BTA6, 10, 12, and 27. The JMJD1C gene is in the CON6 region that accounts for the largest proportion 


\section{CON3}

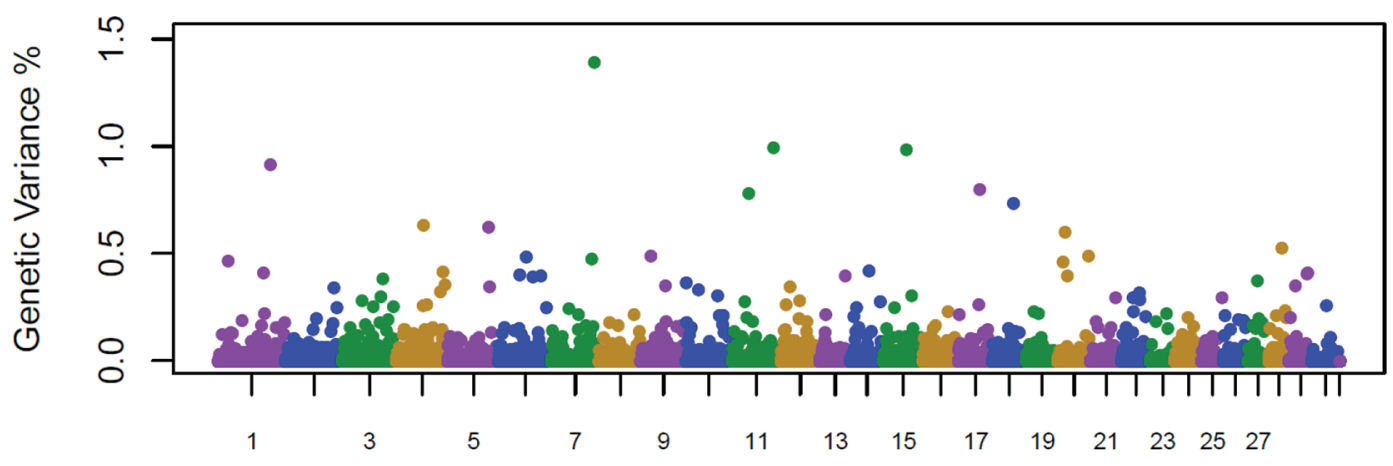

RESP3

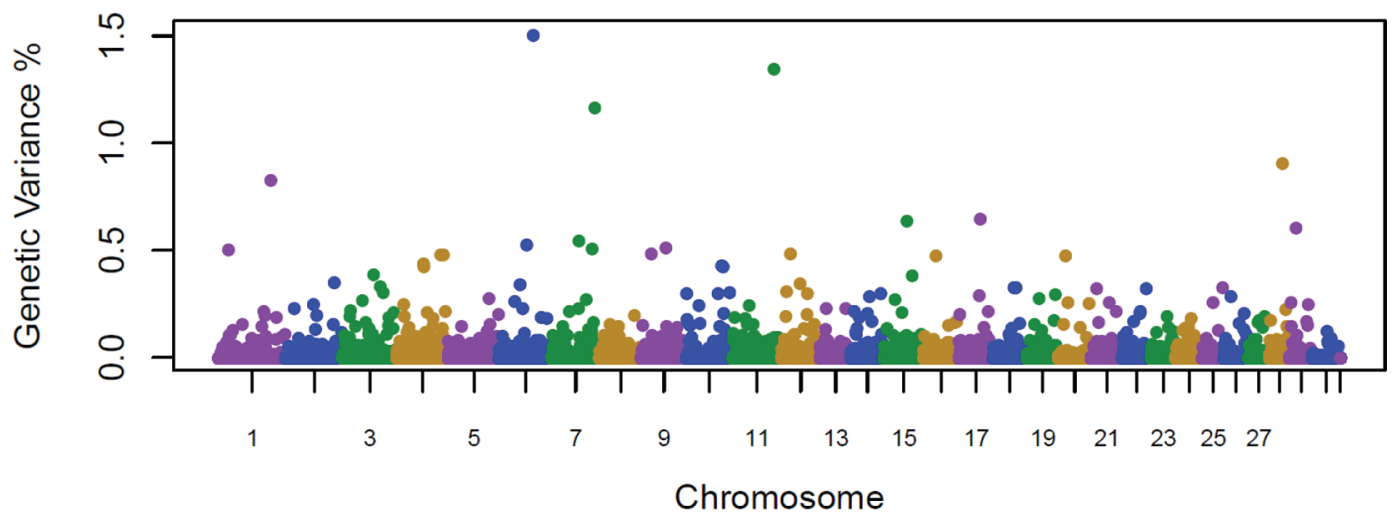

Figure 1. Manhattan plots of the proportion of variance explained by 1-Mb non-overlapping windows for lung consolidation $\{$ top panel: CON3, defined as lung ultrasound (LU) positive $[\mathrm{LU}(+)]$ as affected vs. all others as healthy \} and bovine respiratory disease \{bottom panel: RESP3, defined as clinical respiratory scoring (CRS) positive $[\mathrm{CRS}(+)]$ or $\mathrm{CRS}(+)$ and $\mathrm{LU}(+)$ as affected vs. all others as healthy 3 at 3 wk of age.

of variance $(0.95 \%$ of genetic variance), BTA28 from $19,115,046$ to $20,114,457$. This gene has been associated with chamber-dependent pre-disposition of ventricular myocardium and the differential response of pressure and volume overloads after birth in piglets (Torrado et al., 2011). The regions on BTA12, which explained $0.99 \%$ of the variance for RESP 6 and $0.91 \%$ of the variance for CON6, contain the SLITRK5 gene, which has been associated with left-displaced abomasum pathogenesis in Holstein cattle and other cardiovascular and synaptic functions (Biffani et al., 2014).

Table 2 provides a list of genes in chromosomal regions explaining $\geq 0.7 \%$ of the genetic variance in 3 -wk phenotypes, whereas Table 3 shows genes within the 5 chromosomal regions explaining $\geq 0.7 \%$ of the genetic variance in 6 -wk phenotypes. Prominent genes related to immune function and antiviral response were present, such as TRIM21, which was associated with CON3 (Stelzer et al., 2016). In addition, a gene related to ulcer occurrence and distal muscle weakness (SPTLC2) was observed in the regions with large proportion of variance for RESP6 and CON6 (Stelzer et al., 2016).
Overall, the CON3 and CON6 phenotypes tended to be more strongly associated with regions containing lung and respiratory related genes, whereas RESP3 and RESP6 phenotypes were more closely associated with genes related to growth and metabolism.

As noted earlier, Neibergs et al. (2014) carried out a GWAS for BRD in Holstein calves in a case-control study in California and New Mexico, where phenotypes represented a combination of clinical respiratory scores and deep nasopharyngeal swabs and mid-nasal diagnostic swabs. Proportions of variance attributed to $1-\mathrm{Mb}$ regions that were in close proximity to those implicated in the present study included: BTA6 $(95-96 \mathrm{Mb})$ in the California herds and BTA12 $(58-59 \mathrm{Mb})$ in the California and New Mexico herds. Genomic regions explaining the largest proportions of variance in Neibergs et al. (2014) spanned genes including PVRL1 (mediates entry of BHV-1), AZIN1 (expressed in lung and skeletal muscle), and SLIT3 (many functions, including a role in inflammation).

Keele et al. (2015) reported 14 SNP that were significantly associated with the presence of severe lung 
Table 2. Chromosomal regions explaining the largest proportion of genetic variance in 3-wk bovine respiratory disease (BRD) phenotypes, as well as putative genes located in these regions, where RESP3 $=3$-wk-old calves clinical respiratory scoring $($ CRS $)$ positive $[$ CRS $(+)]$ or CRS $(+)$ and lung ultrasound $(\mathrm{LU})$ positive $[\mathrm{LU}(+)]$ were considered $\mathrm{BRD}(+)$ (affected), else healthy; $\mathrm{CON} 3=$ calves $\mathrm{LU}(+)$ were considered $\mathrm{BRD}(+)$ (affected), else healthy ${ }^{1}$

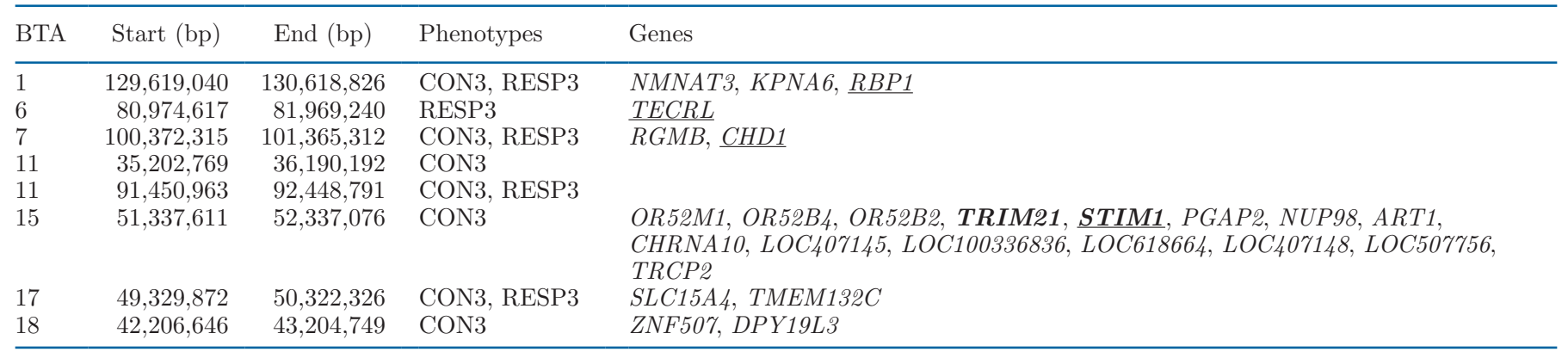

${ }^{1}$ Bolded genes are related to respiratory functions; underlined genes are related to growth functions.

lesions in feedlot beef cattle at slaughter; these were located near genes involved in tissue regeneration (FJX1) and regulation of alternative splicing of pulmonary-related genes (SRRM1 regulates SD44). None of the significant SNP in Keele et al. (2015) were located within regions implicated in the present study, perhaps due to differences in breed, age at measurement, and diagnostic method. Postmortem Kosher lung evaluation phenotypes for male Holstein calves were used in a GWAS of susceptibility to BRD in Israel (Lipkin et al., 2016), and important genomic regions were identified based on sliding $100-\mathrm{Kb}$ windows. Although genomic regions and putative candidate genes of Lipkin et al. (2016) did not overlap with the present study, genes
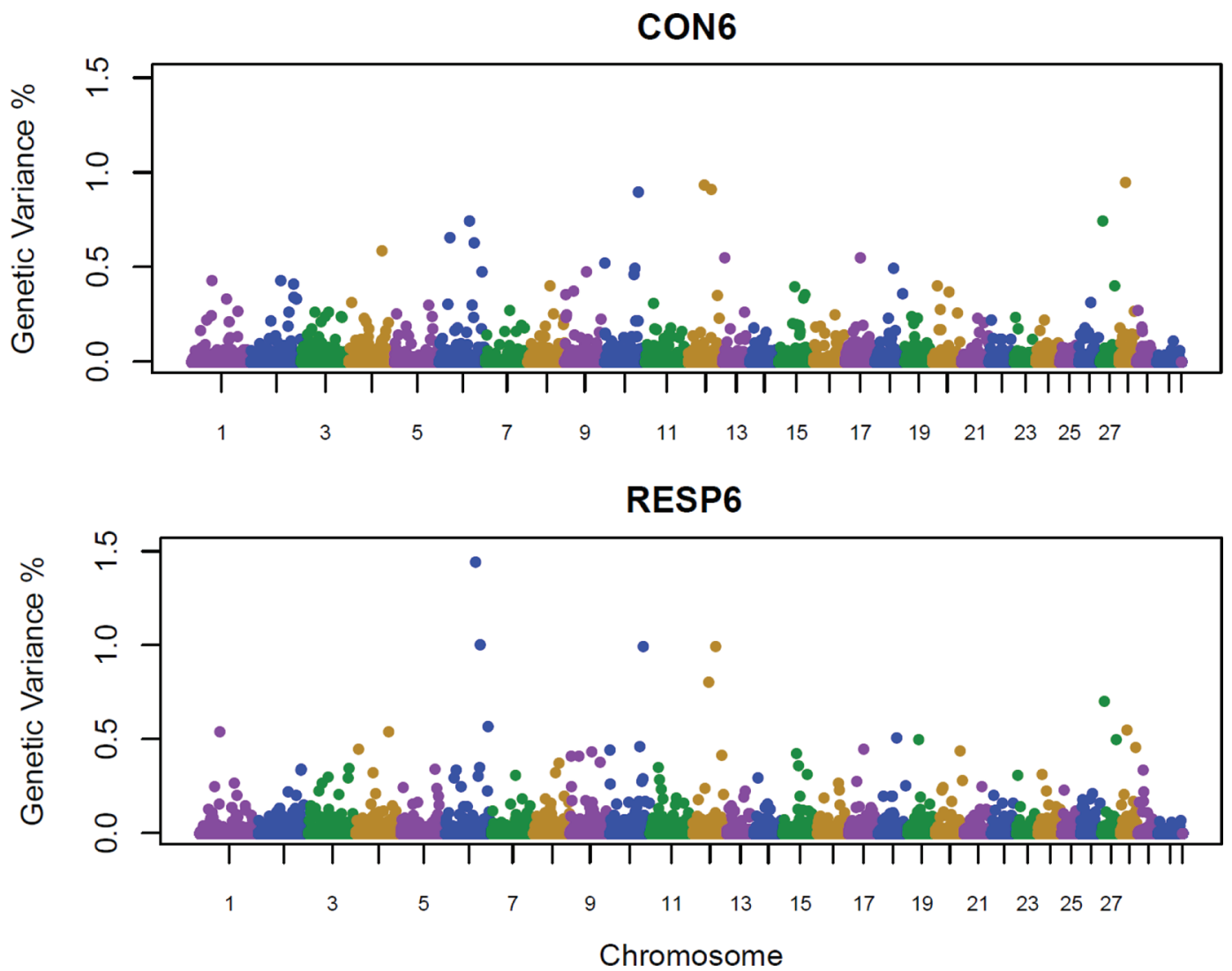

Figure 2. Manhattan plots of the proportion of variance explained by 1-Mb non-overlapping windows for lung consolidation \{top panel: CON6, defined as lung ultrasound (LU) positive $[\mathrm{LU}(+)]$ as affected vs. all others as healthy\} and bovine respiratory disease $\{$ bottom panel: RESP6, defined as clinical respiratory scoring (CRS) positive $[\mathrm{CRS}(+)]$ or $\mathrm{CRS}(+)$ and $\mathrm{LU}(+)$ as affected vs. all others as healthy at 6 wk of age. 
identified in that study had functions related to immunity or structure, such as CADM2 (adhesive activity) and SMOC2 (wound repair).

Farmer-reported health events were used in 2 recent studies of BRD susceptibility in dairy calves (Mahmoud et al., 2017; Vukasinovic et al., 2018). In the German study of Mahmoud et al. (2017), phenotypes were categorized as general disease, diarrhea, or respiratory disorder during the period from birth to 2 mo of age. Markers associated with respiratory disease in calves were identified on BTA8 and 19; in the same study, associations between SNP and respiratory disease in first lactation cows were found on BTA7, 8, and 23.

Most recently, Vukasinovic et al. (2018) used approximately 3 million farmer-reported respiratory disorder events of Holstein calves, of which more than 292,000 had been genotyped, in a ssGWAS analysis with sliding 10-SNP windows. Although no genomic regions overlapped with those identified in the present study, several regions contributed large proportions of variance, including BTA1 (127,288,890 to $127,426,647$ bp) and BTA27 (2,021,945 to $2,342,720 \mathrm{bp} ; 4,456,590$ to $4,476,626 \mathrm{bp} ; 19,087,624$ to $19,195,734 \mathrm{bp}$ ) in calves 4 to $14 \mathrm{~d}$ of age, as well as BTA9 $(43,616,136$ to $43,960,964 \mathrm{bp})$ and BTA14 (11,490,338 to $11,816,360$ $\mathrm{bp}$ ) in calves 15 to $50 \mathrm{~d}$ of age. Two regions of the $\mathrm{X}$ chromosome $(109,250,657$ to $109,734,240 \mathrm{bp}$; $126,011,140$ to $127,636,818$ bp) were associated with BRD phenotypes in Vukasinovic et al. (2018). Because many genes involving growth and cell differentiation (e.g., IGF2 and $Z F X$ ) were implicated, the authors concluded that respiratory disorders may be related to body size. Several genomic regions identified in the present study also contained genes with known roles in growth and related functions (e.g., TECRL, STIM1, and NUP98). A recent study found that calves with lung consolidation had decreased ADG (Cramer and Ollivett, 2019). This study assists in providing context to genes related to body growth and their associations with BRD. Additionally, there were genes in the pres- ent study that were associated with immunity (e.g., TRIM21 and NUP98). Calves between 3 and 5 wk of age tend to have increased prevalence of disease in this time-frame, as the window of susceptibility is present (the transition a calf experiences as passive immunity wears off and active immunity increases; Chase et al., 2008).

\section{Estimated Genetic Parameters}

Estimates of genetic and residual variance components and heritability parameters are provided in Table 4. Phenotypes evaluated at $3 \mathrm{wk}$ of age yielded higher heritability estimates, at 0.21 for CON3 and 0.24 for RESP3, as compared with those evaluated at 6 wk of age, which were 0.08 for CON6 and 0.11 for RESP6. These results seem to indicate that a calf's susceptibility to infection and disease progression may be more heavily influenced by its genetic predisposition at 3 wk of age, whereas environmental conditions related to housing, nutrition, and pathogen exposure may play a greater role at 6 wk of age in the face of waning maternal antibody (Chase et al., 2008). The marked difference in heritability estimates at 3 versus 6 wk of age reflected a large decrease in genetic variance and modest increase in residual variance for both traits. Age of calf appeared to affect heritability estimates in Vukasinovic et al. (2018) as well, where a heritability estimate of 0.097 was reported for the youngest calves ( 0 to $3 \mathrm{~d}$ of age), and heritability estimates decreased steadily to 0.039 for the oldest calves (51 to $365 \mathrm{~d}$ of age).

In the present study, heritability estimates for RESP3 and RESP6 were slightly higher than those of CON3 and CON6. That said, relatively few calves were classified with upper respiratory tract infections (2.9 and $2.7 \%$ at 3 and 6 wk of age, respectively), so differences in genetic parameters were small between disease classification. Heritability estimates from the present study were within the range of estimates reported previously

Table 3. Chromosomal regions explaining the largest proportion of genetic variance in 6-wk bovine respiratory disease (BRD) phenotypes, as well as putative genes located in these regions, where RESP $6=6$-wk-old calves clinical respiratory scoring $(\mathrm{CRS})$ positive $[\mathrm{CRS}(+)]$ or $\mathrm{CRS}(+)$ and lung ultrasound $(\mathrm{LU})$ positive $[\mathrm{LU}(+)]$ were considered $\mathrm{BRD}(+)$ (affected), else healthy; CON6 = 6-wk-old calves LU $(+)$ were considered $\mathrm{BRD}(+)$ (affected), else healthy

\begin{tabular}{lrrll}
\hline BTA & Start $(\mathrm{bp})$ & End $(\mathrm{bp})$ & Phenotypes & Genes \\
\hline 6 & $80,974,617$ & $81,969,240$ & RESP6, CON6 & TECRL \\
6 & $93,025,148$ & $94,020,625$ & RESP6 & CCDC158, SHROOM3, SOWAHB, SEPT11, CCNI, CCNG2 \\
10 & $89,535,705$ & $90,535,118$ & CON6, RESP6 & NGB, POMT2, GSTZ1, TMED8, SAMD15, NOXRED1, U6atac, VIPAS39, AHSA1, \\
& & & & ISM2, SPTLC2, ALKBH1, SNW1, ADCK1 \\
12 & $41,197,611$ & $42,188,688$ & CON6, RESP6 & \\
12 & $62,332,449$ & $63,331,829$ & CON6, RESP6 & SLITRK5 \\
27 & $7,034,011$ & $8,029,701$ & CON6, RESP6 & VEGFC, NEIL3, AGA \\
28 & $19,115,046$ & $20,114,457$ & CON6 & NRBF2, JMJD1 C, REEP3 \\
\hline
\end{tabular}

${ }^{1}$ Bolded genes are related to respiratory functions; underlined genes are related to growth functions. 
in dairy calves (Neibergs et al., 2014) and beef steers (Snowder et al., 2005). Heritability estimates for calf BRD from Vukasinovic et al. (2018), which ranged from 0.039 to 0.097 , were similar to estimates for other farmer-reported health events, such as calf scours (0.045), calf bloat (0.04), mastitis (0.06), ketosis (0.09), and metritis (0.04; Henderson et al., 2011; Parker Gaddis et al., 2014). However, it is known that more accurate characterization of phenotypes can provide higher heritability estimates, and estimates obtained in the present study ( 0.21 to 0.24 at 3 wk of age and 0.08 to 0.11 at 6 wk of age) were substantially higher than those from analyses that relied on farmer-reported health events. For this reason, it appears that the additional investment in labor and equipment for assessment of calves with LU is justified.

\section{Genetic Correlations by Age and Trait Definition}

Genetic parameters from the pairwise multiple-trait analyses of RESP3 and CON3, RESP6 and CON6, RESP3 and RESP6, and CON3 and CON6 are shown in Table 5. As expected, heritability estimates from the multiple-trait models were very consistent with the aforementioned estimates from the single-trait models. Genetic correlations of RESP3 with CON3 and RESP6 with CON6 were extremely high, at 0.98 and 0.99 , respectively. These results are not surprising, given the very slight difference in trait definitions. Differences by age were larger, though, with genetic correlations of 0.66 for RESP3 with RESP6 and 0.69 for CON3 with CON6. These results indicate that respiratory disease at 3 wk of age may be a slightly different trait than respiratory disease at $6 \mathrm{wk}$ of age, possibly because calves are exposed to a broader array of pathogens and environmental challenges over time. To the authors' knowledge, no previous studies have looked at the genetic correlations of ultrasound-based phenotypes between calves of different ages.

\section{GEBV}

The standard deviations of GEBV for RESP3, CON3, RESP6, and CON6 were 0.010, 0.087, 0.053, and 0.040, respectively, indicating considerable genetic variation and potential for improving the respiratory health of dairy calves by genomic selection. Average reliabilities of predicted GEBV were $30 \%$ for RESP3, $27 \%$ for CON3, $16 \%$ for RESP6, and $12 \%$ for CON6, reflecting differences in the heritability estimates. Although these values are low, as compared with traits such as milk yield, SCS, or linear type traits, they were derived from a very small reference population of 1,014 calves with genotypes and BRD phenotypes. Vukasinovic et
Table 4. Genetic $(g)$ and residual $(e)$ variance components and their $\mathrm{SE}$, along with estimated $\mathrm{h}^{2}$, for lung consolidation at $3 \mathrm{wk}$ of age (CON3), bovine respiratory disease at 3 wk of age (RESP3), lung consolidation at $6 \mathrm{wk}$ of age $(\mathrm{CON} 6)$, and bovine respiratory disease at 6 wk of age (RESP6)

\begin{tabular}{lccc}
\hline Phenotype & $\sigma_{g}^{2}(\mathrm{SE})$ & $\sigma_{e}^{2}(\mathrm{SE})$ & $\mathrm{h}^{2}$ \\
\hline CON3 & $0.027(0.010)$ & $0.100(0.010)$ & 0.21 \\
RESP3 & $0.036(0.013)$ & $0.112(0.012)$ & 0.24 \\
CON6 & $0.012(0.010)$ & $0.136(0.011)$ & 0.08 \\
RESP6 & $0.018(0.011)$ & $0.144(0.012)$ & 0.11 \\
\hline
\end{tabular}

al. (2018) reported a mean reliability of $42 \%$ of GEBV for BRD at 25 to $50 \mathrm{~d}$ of age for genotyped animals without phenotypes or progeny, based on 633,123 phenotypic records with farmer-reported health events. Measurement of thousands of additional calves using CRS and LU would be needed before implementation of a routine genomic selection program to reduce the incidence of BRD in the national dairy population using the strategy described herein.

Information about the genetic predisposition of individual animals to BRD can also be used to develop genome-guided management strategies to improve the respiratory health of dairy calves. In order for such programs to be useful in practice, farmers would need to receive genomic predictions for their calves within the first $2 \mathrm{wk}$ of life, so preventative measures could be taken for the most susceptible calves. This may require farmers to send in samples earlier and more frequently, as well as faster turn-around in the genotyping laboratories. In the near term, development of a genomic selection program appears justified. The authors suggest using RESP3 in selection and management decisions regarding calf respiratory health, due to higher prevalence rate, ability to accommodate both clinical and subclinical disease, and greater heritability and reliability estimates. In the future, with accurate knowledge about each animal's genomic predisposition to BRD from a larger reference population, young bulls entering $\mathrm{AI}$ programs could be screened for expected BRD

Table 5. Heritability estimates and genetic correlations $\left(\mathrm{r}_{\mathrm{g}}\right)$ from the pairwise multiple-trait models: bovine respiratory disease at $3 \mathrm{wk}$ of age and lung consolidation at 3 wk of age (RESP3, CON3), bovine respiratory disease at $6 \mathrm{wk}$ of age and lung consolidation at $6 \mathrm{wk}$ of age (RESP6, CON6), bovine respiratory disease at 3 and 6 wk of age (RESP3, RESP6), and lung consolidation at 3 and 6 wk of age (CON3, CON6)

\begin{tabular}{lccc}
\hline Trait 1, trait 2 & $\begin{array}{c}\mathrm{h}^{2} \\
\text { (Trait 1) }\end{array}$ & $\begin{array}{c}\mathrm{h}^{2} \\
\text { (Trait 2) }\end{array}$ & $\begin{array}{c}\mathrm{r}_{\mathrm{g}} \\
\text { (Trait 1, trait 2) }\end{array}$ \\
\hline RESP3, CON3 & 0.24 & 0.22 & 0.98 \\
RESP6, CON6 & 0.11 & 0.10 & 0.99 \\
RESP3, RESP6 & 0.25 & 0.12 & 0.66 \\
CON3, CON6 & 0.22 & 0.09 & 0.69 \\
\hline
\end{tabular}


susceptibility of their offspring. Predictions for BRD susceptibility could be incorporated into the Lifetime Net Merit index (Council on Dairy Cattle Breeding, Bowie, MD), with economic weights that correspond to the prevalence and cost of this disease in the national dairy population.

\section{CONCLUSIONS}

In this study, a practical and feasible protocol determined BRD phenotypes in dairy calves using CRS and lung ultrasonography. Pairs of trained evaluators can assign clinical scores and carry out ultrasound scans on 50 to 100 calves per day on large dairy farms or calf ranches. This protocol should facilitate rapid establishment of large genomic reference populations for the major dairy cattle breeds. Whole-genome selection, or selection focused on specific candidate genes identified by GWAS, would provide increased resistance to bovine respiratory disease during the pre-weaning period, which would in turn reduce the severity of lung damage and mitigate its effect on future performance. Heritability estimates for BRD derived from CRS and LU examination were low to moderate, but substantially higher than previously reported estimates from farmerreported health events. Significant genetic variation between individuals and families, indicating that genetic selection for animals that are more resistant to BRD infection and more resilient to development of subclinical or clinical lung lesions is likely to be effective. Reliabilities of genomic predictions were low, due to the small reference population, but the protocol for measurement of BRD phenotypes proposed in this study can easily be applied to rapidly evaluate thousands or tens of thousands of additional calves to build a sizable and effective reference population. Future studies are needed to confirm genomic regions and putative genes implicated in the present study, including candidate genes with functions related to respiration and immunity; additionally, future work should focus on developing strategies to train evaluators and routinely evaluate large numbers of calves on commercial farms and calf ranches, with particular focus on elite young males and females in the nucleus herds of AI companies.

\section{ACKNOWLEDGMENTS}

This project was supported by USDA Hatch Grant 142-AAA3859. A. Quick acknowledges the cooperating dairy farms for participating in the study, the assistance of Richard Wallace (Zoetis Inc.) in identifying potential farms, and the assistance of undergraduate researchers (Meghan Skiba, Alyssa Nuttleman, and Darby Weigel, University of Wisconsin, Madison) in data collection.

\section{REFERENCES}

Aguilar, I., I. Misztal, D. L. Johnson, A. Legarra, S. Tsuruta, and T. J. Lawlor. 2010. Hot topic: a unified approach to utilize phenotypic, full pedigree, and genomic information for genetic evaluation of Holstein final score. J. Dairy Sci. 93:743-752. https://doi.org/10 $.3168 /$ jds.2009-2730.

Biffani, S., N. Morandi, V. Locatelli, D. Pravettoni, A. Boccardo, A. Stella, E. L. Nicolazzi, and F. Biscarini. 2014. Adding evidence for a role of the SLITRK gene family in the pathogenesis of left displacement of the abomasum in Holstein-Friesian dairy cows. Livest. Sci. 167:104-109. https://doi.org/10.1016/j.livsci.2014.05.002.

Browning, B. L., and S. R. Browning. 2016. Genotype imputation with millions of reference samples. Am. J. Hum. Genet. 98:116-126. https://doi.org/10.1016/j.ajhg.2015.11.020.

Buchanan, J. W., M. D. Macneil, R. C. Raymond, A. R. McClain, and A. L. Van Eenennaam. 2016. Rapid communication: Variance component estimates for Charolais-sired fed cattle and relative economic impact of bovine respiratory disease. J. Anim. Sci 94:5456-5460. https://doi.org/10.2527/jas.2016-1001.

Buczinski, S., G. Forté, and A. M. Bélanger. 2013. Short communication: Ultrasonographic assessment of the thorax as a fast technique to assess pulmonary lesions in dairy calves with bovine respiratory disease. J. Dairy Sci. 96:4523-4528. https://doi.org/10.3168/jds .2013-6577.

Buczinski, S., G. Forté, D. Francoz, and A. M. Bélanger. 2014. Comparison of thoracic auscultation, clinical score, and ultrasonography as indicators of bovine respiratory disease in preweaned dairy calves. J. Vet. Intern. Med. 28:234-242. https://doi.org/10.1111/ jvim.12251.

Buczinski, S., T. L. Ollivett, and N. Dendukuri. 2015. Bayesian estimation of the accuracy of the calf respiratory scoring chart and ultrasonography for the diagnosis of bovine respiratory disease in pre-weaned dairy calves. Prev. Vet. Med. 119:227-231. https://doi .org/10.1016/j.prevetmed.2015.02.018.

Chase, C. C. L., D. J. Hurley, and A. J. Reber. 2008. Neonatal immune development in the calf and its impact on vaccine response. Vet. Clin. North Am. Food Anim. Pract. 24:87-104. https://doi.org/10 .1016/j.cvfa.2007.11.001.

Cramer, M., and T. Ollivett. 2019. Growth of preweaned, group-housed dairy calves diagnosed with respiratory disease using clinical respiratory scoring and thoracic ultrasound - A cohort study. J. Dairy Sci. 102:4322-4331. https://doi.org/10.3168/jds.2018-15420.

de Almeida Santana, M. H., G. A. O. Junior, A. S. M. Cesar, M. C. Freua, R. da Costa Gomes, S. da Luz e Silva, P. R. Leme, H. Fukumasu, M. E. Carvalho, R. V. Ventura, L. L. Coutinho, H. N. Kadarmideen, and J. B. S. Ferraz. 2016. Copy number variations and genome-wide associations reveal putative genes and metabolic pathways involved with the feed conversion ratio in beef cattle. J. Appl. Genet. https://doi.org/10.1007/s13353-016-0344-7.

Dekkers, J. C. M. 1992. Asymptotic response to selection on best linear unbiased predictors of breeding values. Anim. Prod. 54:351360. https://doi.org/10.1017/S0003356100020808.

Dias, M. M., A. Cánovas, C. Mantilla-Rojas, D. G. Riley, P. LunaNevarez, S. J. Coleman, S. E. Speidel, R. M. Enns, A. Islas-Trejo, J. F. Medrano, S. S. Moore, M. R. S. Fortes, L. T. Nguyen, B. Venus, I. S. D. P. Diaz, F. R. P. Souza, L. F. S. Fonseca, F. Baldi, L. G. Albuquerque, M. G. Thomas, and H. N. Oliveira. 2017. SNP detection using RNA-sequences of candidate genes associated with puberty in cattle. Genet. Mol. Res. 16. https://doi.org/10.4238/ gmr16019522.

Dunn, T. R., T. L. Ollivett, D. L. Renaud, K. E. Leslie, S. J. LeBlanc, T. F. Duffield, and D. F. Kelton. 2018. The effect of lung consolidation, as determined by ultrasonography, on first-lactation milk production in Holstein dairy calves. J. Dairy Sci. 101:5404-5410. https://doi.org/10.3168/jds.2017-13870.

Goddard, M. E., and B. J. Hayes. 2007. Genomic selection. J. Anim. Breed. Genet. 124:323-330. https://doi.org/10.1111/j.1439-0388 2007.00702.x.

Gonzalez-Peña, D., N. Vukasinovic, J. J. Brooker, C. A. Przybyla, and S. K. DeNise. 2019. Genomic evaluation for calf wellness traits in 
Holstein cattle. J. Dairy Sci. 102:2319-2329. https://doi.org/10 $.3168 /$ jds.2018-15540.

Gorden, P. J., and P. Plummer. 2010. Control, management, and prevention of bovine respiratory disease in dairy calves and cows. Vet. Clin. North Am. Food Anim. Pract. 26:243-259. https://doi.org/ 10.1016/j.cvfa.2010.03.004.

Gulliksen, S. M., K. I. Lie, and O. Østerås. 2009. Calf health monitoring in Norwegian dairy herds. J. Dairy Sci. 92:1660-1669. https:// doi.org/10.3168/jds.2008-1518.

Henderson, L., F. Miglior, A. Sewalem, J. Wormuth, D. Kelton, A. Robinson, and K. E. Leslie. 2011. Short communication: Genetic parameters for measures of calf health in a population of Holstein calves in New York State. J. Dairy Sci. 94:6181-6187. https://doi .org/10.3168/jds.2011-4347.

Heringstad, B., Y. M. Chang, D. Gianola, and O. Østerås. 2008. Short communication: Genetic analysis of respiratory disease in Norwegian Red calves. J. Dairy Sci. 91:367-370. https://doi.org/10 3168/jds.2007-0365.

Keele, J. W., L. A. Kuehn, T. G. McDaneld, R. G. Tait, S. A. Jones, T. P. L. Smith, S. D. Shackelford, D. A. King, T. L. Wheeler, A. K. Lindholm-Perry, and A. K. McNeel. 2015. Genomewide association study of lung lesions in cattle using sample pooling. J. Anim. Sci. 93:956-964. https://doi.org/10.2527/jas.2014-8492.

Lipkin, E., M. G. Strillacci, H. Eitam, M. Yishay, F. Schiavini, M. Soller, A. Bagnato, and A. Shabtay. 2016. The use of Kosher phenotyping for mapping QTL affecting susceptibility to bovine respiratory disease. PLoS One 11:e0153423. https://doi.org/10.1371/ journal.pone.0153423.

Mahmoud, M., T. Yin, K. Brügemann, and S. König. 2017. Phenotypic, genetic, and single nucleotide polymorphism marker associations between calf diseases and subsequent performance and disease occurrences of first-lactation German Holstein cows. J Dairy Sci. 100:2017-2031. https://doi.org/10.3168/jds.2016-11767.

McGuirk, S. M., and S. F. Peek. 2014. Timely diagnosis of dairy calf respiratory disease using a standardized scoring system. Anim. Health Res. Rev. 15:145-147. https://doi.org/10.1017/ S1466252314000267.

Misztal, I., S. Tsuruta, D. Lourenco, I. Aguilar, A. Legarra, and Z. Vitezica. 2015. Manual for BLUPF90 family of programs. http:// nce.ads.uga.edu/wiki/lib/exe/fetch.php?media=blupf90_all2.pdf.

Neibergs, H. L., C. M. Seabury, A. J. Wojtowicz, Z. Wang, E. Scraggs, J. N. Kiser, M. Neupane, J. E. Womack, A. Van Eenennaam, G. R. Hagevoort, T. W. Lehenbauer, S. Aly, J. Davis, and J. F. Taylor. 2014. Susceptibility loci revealed for bovine respiratory disease complex in pre-weaned Holstein calves. BMC Genomics 15:1164. https://doi.org/10.1186/1471-2164-15-1164.

Ollivett, T. L., and S. Buczinski. 2016. On-farm use of ultrasonography for bovine respiratory disease. Vet. Clin. North Am. Food Anim. Pract. 32:19-35. https://doi.org/10.1016/j.cvfa.2015.09.001.

Ollivett, T. L., J. L. Caswell, D. V. Nydam, T. Duffield, K. E. Leslie, J. Hewson, and D. Kelton. 2015. Thoracic ultrasonography and bronchoalveolar lavage fluid analysis in Holstein calves with subclinical lung lesions. J. Vet. Intern. Med. 29:1728-1734. https://doi .org/10.1111/jvim.13605.

Parker Gaddis, K. L., J. B. Cole, J. S. Clay, and C. Maltecca. 2014. Genomic selection for producer-recorded health event data in US dairy cattle. J. Dairy Sci. 97:3190-3199. https://doi.org/10.3168/ jds.2013-7543.

Purcell, S., B. Neale, K. Todd-Brown, L. Thomas, M. A. R. Ferreira, D. Bender, J. Maller, P. Sklar, P. I. W. De Bakker, M. J. Daly, and P. C. Sham. 2007. PLINK: A tool set for whole-genome association and population-based linkage analyses. Am. J. Hum. Genet. 81:559-575. https://doi.org/10.1086/519795.

Sadkowski, T., M. Jank, L. Zwierzchowski, J. Oprzadek, and T. Motyl. 2009. Comparison of skeletal muscle transcriptional profiles in dairy and beef breeds bulls. J. Appl. Genet. 50:109-123. https:// doi.org/10.1007/BF03195662.

Sivula, N., T. Ames, W. Marsh, and R. Werdin. 1996. Descriptive epidemiology of morbidity and mortality in Minnesota dairy heifer calves. Prev. Vet. Med. 27:155-171. https://doi.org/10.1016/0167 -5877(95)01000-9.

Snowder, G. D., L. D. Van Vleck, L. V. Cundiff, and G. L. Bennett. 2005. Influence of breed, heterozygosity, and disease incidence on estimates of variance components of respiratory disease in preweaned beef calves. J. Anim. Sci. 83:1247-1261.

Stanton, A. L., D. F. Kelton, S. J. LeBlanc, J. Wormuth, and K. E. Leslie. 2012. The effect of respiratory disease and a preventative antibiotic treatment on growth, survival, age at first calving, and milk production of dairy heifers. J. Dairy Sci. 95:4950-4960. https: //doi.org/10.3168/jds.2011-5067.

Stelzer, G., N. Rosen, I. Plaschkes, S. Zimmerman, M. Twik, S. Fishilevich, T. I. Stein, R. Nudel, I. Lieder, Y. Mazor, S. Kaplan, D. Dahary, D. Warshawsky, Y. Guan-Golan, A. Kohn, N. Rappaport, M. Safran, and D. Lancet. 2016. The GeneCards Suite: From gene data mining to disease genome sequence analyses. Curr. Protoc. Bioinformatics 54. https://doi.org/10.1002/cpbi.5.

Teixeira, A. G. V., J. A. A. McArt, and R. C. Bicalho. 2017. Thoracic ultrasound assessment of lung consolidation at weaning in Holstein dairy heifers: Reproductive performance and survival. J. Dairy Sci. 100:2985-2991. https://doi.org/10.3168/jds.2016-12016.

Torrado, M., R. Iglesias, A. Centeno, and A. Mikhailov. 2011. Molecular signature analysis reveals a set of genes that can be involved in balance between concentric and eccentric cardiac growth. Eur. J. Heart Fail. Suppl. 10, S215-S217. https://doi.org/10.1093/eurjhf/ hsr010.

USDA. 2010. Heifer calf health and management practices on U.S. dairy operations, 2007. Fort Collins, CO.

USDA. 2012. An overview of operations that specialize in raising dairy heifers, 2010. Fort Collins, CO.

USDA. 2018. Health and Management Practices on U.S. Dairy Operations, 2014. Fort Collins, CO.

van der Fels-Klerx, H. J., J. T. Sorensen, A. W. Jalvingh, and R. B. M. Huirne. 2001. An economic model to calculate farm-specific losses due to bovine respiratory disease in dairy heifers. Prev. Vet. Med. 51:75-94. https://doi.org/10.1016/S0167-5877(01)00208-2.

Van Vleck, L. D., A. F. Hakim, L. V. Cundiff, R. M. Koch, J. D. Crouse, and K. G. Boldman. 1992. Estimated breeding values for meat characteristics of crossbred cattle with an animal model. J. Anim. Sci. 70:363-371. https://doi.org/10.2527/1992.702363x.

VanRaden, P. M., C. P. Van Tassell, G. R. Wiggans, T. S. Sonstegard, R. D. Schnabel, J. F. Taylor, and F. S. Schenkel. 2009. Invited review: Reliability of genomic predictions for North American Holstein bulls. J. Dairy Sci. 92:16-24. https://doi.org/10.3168/jds .2008-1514.

Vukasinovic, N., D. Gonzalez-Peña, J. J. Brooker, P. Desai, and S. K. Denise. 2018. Exploring genetics underlying respiratory disorders in dairy calves using producer-recorded data. Page 11. 597 in Proceedings of the World Congress on Genetics Applied to Livestock Production.

Wang, H., I. Misztal, I. Aguilar, A. Legarra, R. L. Fernando, Z. Vitezica, R. Okimoto, T. Wing, R. Hawken, and W. M. Muir. 2014. Genome-wide association mapping including phenotypes from relatives without genotypes in a single-step (ssGWAS) for 6-week body weight in broiler chickens. Front. Genet. 5:134. https://doi .org/10.3389/fgene.2014.00134.

Wang, H., I. Misztal, I. Aguilar, A. Legarra, and W. M. Muir. 2012 Genome-wide association mapping including phenotypes from relatives without genotypes. Genet. Res. (Camb.) 94:73-83. https:// doi.org/10.1017/S0016672312000274.

\section{ORCIDS}

Allison E. Quick (1) https://orcid.org/0000-0002-7616-8921 Theresa L. Ollivett @ https://orcid.org/0000-0003-2490-5835 Brian W. Kirkpatrick @ https://orcid.org/0000-0003-2962-3861 Kent A. Weigel ๑ https://orcid.org/0000-0002-2391-6260 